\title{
It takes two to tango
}

One of the main characteristics of malignant glioblastoma is the rapid formation of new, leaky blood vessels, which are responsible for the oedema that contributes to the numerous neurological symptoms associated with this disease. Hence, multiple studies have assessed targeting of the VEGF pathway in patients with glioblastoma. Interestingly, although the antiangiogenic agent bevacizumab is widely used in the USA for the treatment of recurrent glioblastoma-on the basis of promising response rates-to date, no randomized studies have shown a survival benefit in this setting.

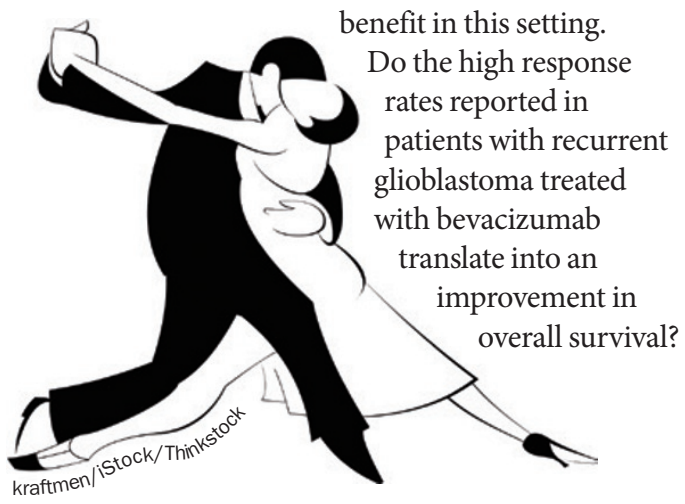

To answer this question, the group led by Van de Bent carried out the BELOB trial, an open-label, multicentre, randomized phase II study.

The trial recruited 153 patients with disease recurrence for the first time after treatment with temozolomide chemoradiotherapy, who were randomly assigned to three treatment arms. The first group received treatment with lomustine administered orally $(n=47)$, the second group received bevacizumab intravenously $(n=51)$ and, finally, the third group received a combination treatment of oral lomustine and intravenous bevacizumab $(n=55)$. The primary end point was overall survival at 9 months based on the median overall survival reported in a previous trial that had assessed bevacizumab versus irinotecan. This end point was reached by $63 \%$ of patients receiving the combination of bevacizumab and lomustine, $43 \%$ of patients in the arm assigned to receive lomustine and $38 \%$ of patients in the group assigned to receive bevacizumab, showing that only the combination of bevacizumab and lomustine reached the efficacy threshold of $55 \%$ overall survival at 9 months. Patients assigned to the two single-agent treatment groups had similar 6-month progressionfree survival (13\% in the lomustine group and $16 \%$ in the bevacizumab group).

After the lomustine dose was reduced in the combination group as a result of the preplanned safety review, the combined treatment was well tolerated. The most frequent grade $\geq 3$ toxic effects were hypertension, fatigue and infections.

The investigators conclude that treatment of recurrent glioblastoma with bevacizumab-but only if combined with lomustine-warrants further investigation in a phase III study.

\section{Teresa Villanueva}

This article is modified from the original in Nat. Rev. Clin. Oncol. (doi:10.1038/nrclinonc.2014.133).

Original article Taal, W. et al. Single-agent bevacizumab or lomustine versus a combination of bevacizumab plus lomustine in patients with recurrent glioblastoma (BELOB trial): a randomised controlled phase 2 trial. Lancet Oncol. doi:10.1016/S1470-2045(14)70314-6 